JOURNAL OF THE

CHUNGCHEONG MATHEMATICAL SOCIETY

Volume 27, No. 1, 2014

http://dx.doi.org/10.14403/jcms.2014.27.1.99

\title{
M-ISOMETRIC WEIGHTED SHIFTS
}

\author{
JUN IK LEE*
}

\begin{abstract}
In this paper, we characterize the m-isometric weighted shifts, using this characterization, we study the relations between the hyponormality and the m-isometricity of operators.
\end{abstract}

\section{Introduction}

Let $\mathcal{H}$ and $\mathcal{K}$ be complex Hilbert spaces, let $\mathcal{L}(\mathcal{H}, \mathcal{K})$ be the set of bounded linear operators from $\mathcal{H}$ to $\mathcal{K}$ and write $\mathcal{L}(\mathcal{H}):=\mathcal{L}(\mathcal{H}, \mathcal{H})$. An operator $T \in \mathcal{L}(\mathcal{H})$ is said to be normal if $T^{*} T=T T^{*}$, hyponormal if $T^{*} T \geq T T^{*}$, and subnormal if $T=\left.N\right|_{\mathcal{H}}$, where $N$ is normal on some Hilbert space $\mathcal{K} \supseteq \mathcal{H}$. If $T$ is subnormal then $T$ is also hyponormal. An operator $T \in \mathcal{L}(\mathcal{H})$ is called an m-isometry if

$$
\sum_{k=0}^{m}(-1)^{k}\left(\begin{array}{c}
m \\
k
\end{array}\right) T^{* k} T^{k}=0 .
$$

It is easy to see that the equation (1.1) is equivalent to the following equation

$$
\sum_{k=0}^{m}(-1)^{k}\left(\begin{array}{c}
m \\
k
\end{array}\right)\left\|T^{k} x\right\|=0
$$

for all $x \in \mathcal{H}$. If $m=1$ then it is said to be isometry. It is easy to see that any $\mathrm{m}$-isometric operator is also $(\mathrm{m}+1)$-isometry. But the converse is not true in general. m-isometric operators was first introduced in $([1],[2],[3])$ and has received much attention in recent years. Given a bounded sequence of positive numbers $\alpha: \alpha_{0}, \alpha_{1}, \cdots$ (called weights),

Received December 11, 2013; Accepted January 16, 2014.

2010 Mathematics Subject Classification: Primary 47B20, 47B37, 47A13, 28A50; Secondary 44A60, 47-04, 47A20.

Key words and phrases: m-isometry, weighted shifts, hyponormal, subnormal, propagation.

*This research was supported by a 2013 Research Grant from Sangmyung University. 
the (unilateral) weighted shift $W_{\alpha}$ associated with $\alpha$ is the operator on $\ell^{2}\left(\mathbb{Z}_{+}\right)$defined by $W_{\alpha} e_{n}:=\alpha_{n} e_{n+1}$ for all $n \geq 0$, where $\left\{e_{n}\right\}_{n=0}^{\infty}$ is the canonical orthonormal basis for $\ell^{2}$. It is straightforward to check that $W_{\alpha}$ can never be normal, and that $W_{\alpha}$ is hyponormal if and only if $\alpha_{n} \leq \alpha_{n+1}$ for all $n \geq 0$. The moments of $\alpha$ are given as

$$
\gamma_{k} \equiv \gamma_{k}(\alpha):=\left\{\begin{array}{cc}
1 & \text { if } k=0 \\
\alpha_{0}^{2} \cdots \alpha_{k-1}^{2} & \text { if } k>0
\end{array}\right.
$$

In this paper, we characterize the m-isometric weighted shifts, using this characterization, we study the relations between the hyponormality and the m-isometricity of operators.

\section{Main results}

We start from a basic result.

Proposition 2.1. Every isometry is subnormal and hence hyponormal.

Proof. If $T \in \mathcal{L}(\mathcal{H})$ is isometry then $T^{*} T=I$. By a direct calculation, we can see that $N:=\left(\begin{array}{cc}T & I-T T^{*} \\ 0 & T^{*}\end{array}\right)$ is a normal extension of $T$. Thus, $T$ is subnormal.

In view of Proposition 2.1, it is interesting to ask that every m-isometric operator is whether subnormal or hyponormal. To answer for this questions, we first give a characterization of m-isometric weighted shifts.

Theorem 2.2. $W_{\alpha}$ is m-isometry if and only if

$$
\sum_{k=0}^{m}(-1)^{k}\left(\begin{array}{c}
m \\
k
\end{array}\right) \gamma_{n+k}=0
$$

for all $n \geq 0$.

Proof. $(\Rightarrow)$ Suppose that $W_{\alpha}$ is m-isometry. Then we have the equation (2.1) from the equation (1.2) taking $x=e_{n}$.

$(\Leftarrow)$ Suppose that the equation $(2.1)$ holds for all $n \geq 0$. Since $W_{\alpha}^{*} W_{\alpha}$ is diagonal, it is easy to show that the equation (1.2) holds for any $x=\sum_{n=1}^{\infty} x_{n} e_{n} \in \mathcal{H}$. Therefore, $W_{\alpha}$ is m-isometry.

Corollary 2.3. For a weighted shift $W_{\alpha}$, we have:

(i) $W_{\alpha}$ is isometry if and only if $\alpha_{n}=1$ for all $n \geq 0$, i.e., $W_{\alpha}$ is the unilateral shift. 
(ii) $W_{\alpha}$ is 2-isometry if and only if $\alpha_{n+1}^{2}=2-\frac{1}{\alpha_{n}^{2}}$ for all $n \geq 0$.

(iii) $W_{\alpha}$ is 3-isometry if and only if $\alpha_{n+2}^{2}=3-\frac{3}{\alpha_{n+1}^{2}}+\frac{1}{\alpha_{n}^{2} \alpha_{n+1}^{2}}$ for all $n \geq 0$.

Proof. (i) If $W_{\alpha}$ is isometry then $\gamma_{n}=\gamma_{n+1}$ for all $n \geq 0$, and hence $\alpha_{n}=1$ for all $n \geq 0$. The converse is clear.

(ii) Note that $W_{\alpha}$ is 2-isometry if and only if $\gamma_{n}-2 \gamma_{n+1}+\gamma_{n+2}=0$ for all $n \geq 0$ if and only if $\alpha_{n+1}^{2}=2-\frac{1}{\alpha_{n}^{2}}$ for all $n \geq 0$.

(iii) Note that $W_{\alpha}$ is 3 -isometry if and only if $\gamma_{n}-3 \gamma_{n+1}+3 \gamma_{n+2}-\gamma_{n+3}=$ 0 for all $n \geq 0$ if and only if $\alpha_{n+2}^{2}=3-\frac{3}{\alpha_{n+1}^{2}}+\frac{1}{\alpha_{n}^{2} \alpha_{n+1}^{2}}$ for all $n \geq 0$.

TheOREM 2.4. If $W_{\alpha}$ is m-isometry and the weight sequence $\alpha$ is convergent, then the limit of $\alpha$ must be 1 .

Proof. Suppose $\lim \alpha_{n}=a$ and $\epsilon>0$ was given. First note that the equation (2.1) is equivalent to

$$
1+\sum_{k=1}^{m}(-1)^{k}\left(\begin{array}{c}
m \\
k
\end{array}\right) \alpha_{n}^{2} \cdots \alpha_{n+k-1}^{2}=0
$$

for all $n \geq 0$. Since $\lim \alpha_{n}=a$, we can see that $\left|1+\sum_{k=1}^{m}(-1)^{k}\left(\begin{array}{c}m \\ k\end{array}\right) a^{2 k}\right|<$ $\epsilon$ for sufficiently large $n$. But since $1+\sum_{k=1}^{m}(-1)^{k}\left(\begin{array}{c}m \\ k\end{array}\right) a^{2 k}=\left(1-a^{2}\right)^{m}$, we have the desired result.

J. Stampfli [8] showed that for subnormal weighted shifts $W_{\alpha}$, a propagation phenomenon occurs which forces the flatness of $W_{\alpha}$ whenever two equal weights are present. Later, A. Joshi proved in [7] that the shift with weights $\alpha_{0}=\alpha_{1}=a, \alpha_{2}=\alpha_{3}=\cdots=b, 0<a<b$, is not quadratically hyponormal, and P. Fan [6] established that for $a=1$, $b=2$, and $0<s<\sqrt{5} / 5, W_{\alpha}+s W_{\alpha}^{2}$ is not hyponormal. On the other hand, it was shown in [5, Theorem 2] that a hyponormal weighted shift with three equal weights cannot be quadratically hyponormal without being flat: If $W_{\alpha}$ is quadratically hyponormal and $\alpha_{n}=\alpha_{n+1}=\alpha_{n+2}$ for some $n \geq 0$, then $\alpha_{1}=\alpha_{2}=\alpha_{3}=\cdots$, i.e., $W_{\alpha}$ is subnormal. Furthermore, in [5, Proposition 11] it was shown that, in the presence of quadratic hyponormality, two consecutive pairs of equal weights again force flatness, thereby subnormality. Y. Choi [4] improved this result, that is, if $W_{\alpha}$ is quadratically hyponormal and $\alpha_{n}=\alpha_{n+1}$ for some $n \geq 1$, then $W_{\alpha}$ is flat. Moreover, Y. Choi [4] also showed that if $W_{\alpha}$ is polynomially hyponormal and $\alpha_{n}=\alpha_{n+1}$ for some $n \geq 0$, then $W_{\alpha}$ is flat. 
Proposition 2.5. (Propagation) Let $W_{\alpha}$ be a weighted shift with weight sequence $\left\{\alpha_{n}\right\}_{n=0}^{\infty}$.

(i) ([8, Theorem 6]) Let $W_{\alpha}$ be subnormal. If $\alpha_{n}=\alpha_{n+1}$ for some $n \geq 0$, then $\alpha$ is flat, i.e., $\alpha_{1}=\alpha_{2}=\alpha_{3}=\cdots$.

(ii) ([5, Corollary 6]) Let $W_{\alpha}$ be 2-hyponormal. If $\alpha_{n}=\alpha_{n+1}$ for some $n \geq 0$, then $\alpha$ is flat.

(iii) ([4, Theorem 1]) Let $W_{\alpha}$ be quadratically hyponormal. If $\alpha_{n}=$ $\alpha_{n+1}$ for some $n \geq 1$, then $\alpha$ is flat.

(iv) ([4, Theorem 2]) Let $W_{\alpha}$ be polynomially hyponormal. If $\alpha_{n}=$ $\alpha_{n+1}$ for some $n \geq 0$, then $\alpha$ is flat.

We now show that 2-isometric weighted shift operator is isometry if two equal weights are presented.

THEOREM 2.6. If $W_{\alpha}$ is 2-isometry with $\alpha_{n}=\alpha_{n+1}$ for some $n \geq 0$ then $\alpha_{n}=1$ for all $n \geq 0$ i.e., $W_{\alpha}$ is isometry.

Proof. By Corollary 2.3 (ii), we can see that $\alpha_{n}=\alpha_{n+1}=1$. Thus, by again Corollary 2.3 (ii), we have $\alpha_{n}=1$ for all $n \geq 0$.

THEOREM 2.7. If $W_{\alpha}$ is 2-isometry then the weight sequence $\alpha$ is decreasing and hence converges to 1.

Proof. If $W_{\alpha}$ is 2-isometry, observe that $\alpha_{n+1}^{2}=2-\frac{1}{\alpha_{n}^{2}} \leq \alpha_{n}^{2}$ for all $n \geq 0$. By the Monotone Convergence Theorem, the weight sequence $\alpha$ converges. By Theorem 2.4 the limit should be 1 .

Corollary 2.8. For a weighted shift $W_{\alpha}$, if $\alpha_{n}<1$ for some $n \geq 0$ then $W_{\alpha}$ is not 2-isometry.

Proof. Since the weight sequence $\alpha$ is decreasing, if $\alpha_{n}<1$ for some $n \geq 0$, then the weight sequence $\alpha$ cannot converge to 1 . Thus, $W_{\alpha}$ is not 2-isometry.

Now, we can give an example which is 2-isometry but not hyponormal( and hence not subnormal).

EXAMPLE 2.9. Let $W_{\alpha}$ be the weighted shift with the weight sequence $\alpha \equiv \sqrt{\frac{n+2}{n+1}}$. Then $W_{\alpha}$ is 2-isometry but not hyponormal.

Proof. Since $\gamma_{n}=n+1$ for all $n \geq 0$, we have $\sum_{k=0}^{2}(-1)^{k}\left(\begin{array}{l}2 \\ k\end{array}\right) \gamma_{n+k}=$ $\gamma_{n}-2 \gamma_{n+1}+\gamma_{n+2}=n+1-2(n+2)+n+3=0$. Thus, $W_{\alpha}$ is 2-isometry. However, $W_{\alpha}$ is not hyponormal because the weights are decreasing. 


\section{References}

[1] J. Agler and M. Stankus, m-Isometric transformation of Hilbert space, I, Integral Equations Operator Theory 21 (1995), 383-429.

[2] J. Agler and M. Stankus, m-Isometric transformation of Hilbert space, II, Integral Equations Operator Theory 23 (1995), 1-48.

[3] J. Agler and M. Stankus, m-Isometric transformation of Hilbert space, III, Integral Equations Operator Theory 24 (1996), 379-421.

[4] Y. B. Choi, A propagation of quadratically hyponormal weighted shifts, Bull. Korean Math. Soc. 37 (2000), no. 2, 347-352.

[5] R. E. Curto, Quadratically hyponormal weighted shifts, Integral Equations Operator Theory, 13 (1990), 49-66.

[6] P. Fan, A note on hyponormal weighted shifts. Proc. Amer. Math. Soc. 92 (1984), 271-272.

[7] A. D. Joshi, An example of a monotone shift which is not quadratically hyponormal. Math. Student 51 (1983), 193-194.

[8] J. Stampfli, Which weighted shifts are subnormal?, Pacific J. Math. 17 (1966), 367-379.

Department of Mathematics Education

Sangmyung University

Seoul 110-743, Republic of Korea

E-mail: jilee@smu.ac.kr 